\title{
Nod to Auth: Fluent AR/VR Authentication with User Head-Neck Modeling
}

\author{
Xue Wang \\ Cornell University \\ Ithaca, NY, USA \\ xw526@cornell.edu
}

\author{
Yang Zhang \\ Carnegie Mellon University \\ Pittsburgh, PA, USA \\ yang.zhang@cs.cmu.edu
}

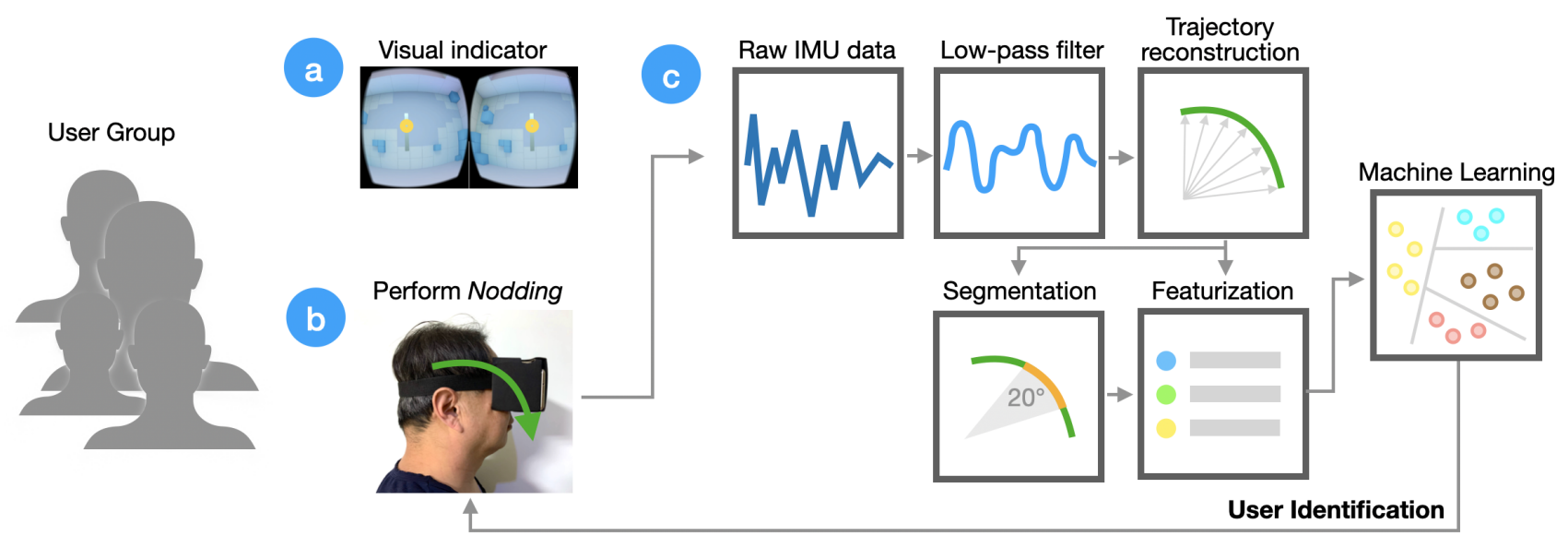

Figure 1: Nod to Auth user identification pipeline.

\begin{abstract}
With AR/VR devices becoming increasingly common around us, their user authentication has posed a critical challenge. While typing passwords is straightforward with a keyboard, it has been cumbersome with conventional AR/VR input techniques such as in-air gestures and hand-held controllers. In this work, we developed a fluent authentication technique that allows AR/VR users to unlock their profiles with simple head gestures (e.g., nodding). This resembles the powerful "Slide to Unlock" interaction on touch screen devices. Specifically, we extract bio-features such as neck length and head radius from IMU sensor readings of these head gestures for user identification with machine learning. Though our approach is less strict compared with conventional passwordbased methods, we believe its swiftness greatly facilitates scenarios with frequent user switching (e.g., device sharing across team and family members) which demand quick authentications. Through a 10-participant evaluation, we demonstrated that our system is robust and accurate with an average accuracy of $97.1 \%$ on groups of 5 , simulating family and lab use.
\end{abstract}

This work is licensed under a Creative Commons Attribution International 4.0 License.

CHI '21 Extended Abstracts, May 8-13, 2021, Yokohama, Japan

(c) 2021 Association for Computing Machinery.

ACM ISBN 978-1-4503-8095-9/21/05.

https://doi.org/10.1145/3411763.3451769

\section{CCS CONCEPTS}

- Human-centered computing $\rightarrow$ Virtual reality.

\section{KEYWORDS}

AR/VR, Authentication, IMUs, Slide to Unlock, Natural Interaction ACM Reference Format:

Xue Wang and Yang Zhang. 2021. Nod to Auth: Fluent AR/VR Authentication with User Head-Neck Modeling. In CHI Conference on Human Factors in Computing Systems Extended Abstracts (CHI '21 Extended Abstracts), May 8-13, 2021, Yokohama, Japan. ACM, New York, NY, USA, 7 pages. https: //doi.org/10.1145/3411763.3451769

\section{INTRODUCTION}

With the emerging success of AR/VR devices, their authentication techniques have received an increasing amount of research effort. Password-based authentication approaches have mostly relied on external input devices which can be cumbersome to carry around and use. Additionally, unlike smartphones and wearables, AR/VR headsets are often shared across users (e.g., entire family sharing one VR headset), making their authentication more frequent than that of personal devices. All of these demand fluent authentication approaches for AR/VR which users could perform swiftly with little interaction overhead.

In this research, we propose Nod to Auth, a novel AR/VR authentication technique that allows users to unlock their headsets by one-stroke gestures as simple as nodding. Our approach is built around IMU sensors featured on most AR/VR devices, which means our approach can be easily and cheaply scaled to a wide variety 
of devices from hobbyists' toys to high-end commodity devices. Specifically, we utilize the IMU sensor to extract biometric features of its wearer's head-neck section. Such features include average neck length, changes in neck length and head orientation during head gestures, and characteristics of the head gesture trajectory, which capture minor but distinctive patterns differing across users. We then use a standard machine learning model to identify users, which according to a 10-participant evaluation, achieves an average accuracy of $97.1 \%, 97.7 \%, 98.5 \%$, and $99.1 \%$ for group sizes of 5, 4, 3, and 2.

\section{RELATED WORK}

\subsection{Human Head-Neck Modeling}

The human head-neck section comprises seven cervical vertebrae connecting via rotational joints [7]. In early studies, the neck was modeled as a continuum beam with straight, elastic, and homogeneous materials while the head was usually treated as a rigid body [14]. With the aid from computers, researchers have developed models that are more sophisticated and accurate. Three types of models are usually used for describing head-neck kinematics: two-pivot models [10], discrete parameter models [26], and finite element models [29]. The two-pivot model presented by Wismans et al. [30] simplifies the head-neck-torso as a three-segment two-joint model, modeling the neck as a rigid or scalable link connecting head and torso. In the discrete parameter model, head and vertebrae are represented by ten rigid bodies, using linear viscoelastic elements to represent the intervertebral soft tissues and muscles. The most complex one is the finite element model $[15,29]$. It describes cervical spine geometry and material behavior in detail with high complexity and computational redundancy.

\subsection{User Identification Based on Bio-features}

Fluent user identification has long been sought after - many previous system leveraged sensors deployed in environments [2, 24, 27]. In this section, we focus on mobile solutions which are more related to Nod to Auth. On this front, high-end commodity mobile devices have included biometric sensors such as Face ID and fingerprint sensors. In the research domain, BiLock [35] leverages dental occlusion differences across users which manifest on characteristic sounds of teeth clicking. CapAuth [12] recognizes users by their hand shapes on touchscreens. Bio-impedance differences across user forearms have also been used for identification [5]. Another common approach recognizes users by extracting their motion characteristics with IMU sensors. People leveraged built-in IMU sensors on smartphones to gather motion data from their smartphone uses [4] and from various implicit activities [9, 17]. It is also possible to leverage custom-built wearable sensors on user fingers [31].

\subsection{AR/VR Authentication}

Previous systems looked into techniques that replaced password typing with natural interactions. For example, LookUnlock [11] allows users to input passwords by looking at virtual objects in an encoded sequence. It is also possible to recognize users based on their writing patterns [28]. Closer to our technique is prior work that extracts kinesiological characteristics from body motions (e.g., head, hand, and torso). For example, identifiable signals can be extracted from physical tasks that users perform in VR environments, such as pointing, grabbing, walking, and typing [19, 20,22]. Our work was directly inspired by these systems and was aimed for interactions that involve only head movements and built-in IMU sensors on AR/VR headsets to eliminate the need for external sensors. As a result, our technique can be readily adopted by a wide array of existing devices.

Closest to our work is Headbanger [18], which leverages distance computing algorithms including cosine, correlation, and DTW in two-label classification tasks to identify users (i.e., true vs. false subject). Headbanger requires users to perform multiple repetitive head gestures along with audio stimuli, taking from 5 seconds to 10 seconds to complete one gesture. In comparison, by leveraging biometric features derived from user head-neck modeling, Nod to Auth requires users to perform head gestures with a single stroke, enabling a swift authentication similar to the phenomenal "Slide to Unlock" technique on touchscreen devices.

\section{SENSING PRINCIPLE}

Human head-neck section is complex and has many joints. Figure 2 left shows its anatomical features and key components. As a compromise to the constrained sensor type and location, our system adopts a ball-stick model which has only one joint (see Figure 2 middle). With this model, the entire system can be determined by angular motion parameters [16], which can be measured by IMU sensors from a single location (i.e., AR/VR headset). Head-neck motions are typically decomposed into three characteristics: 1) initial-end postures, 2) skeletal proportions, and 3) gesture dynamics [32]. Instead of relying solely on gesture dynamics, as shown in prior work, our system also incorporated the other two characteristics. Specifically, we designed features around all characteristics that are consistent when a same user performs the same gesture from time to time. Section 6.3 describes these features in detail.

\section{HEAD GESTURES DESIGN}

We set out to include only one-stroke gestures - those users need to perform only once, same as "Slide to Unlock". Our head gesture set was drawn from prior work on head gesture interactions [18, 20,33] and literature on head-neck biomechanics [23]. Figure 2 right shows the three gestures supported by Nod to Auth, including 1) nodding, 2) turning, and 3) tilting. Each gesture requires users to perform the motion starting from one end and ending at the other, and our system supports both directions. Similar with "Slide to Unlock",
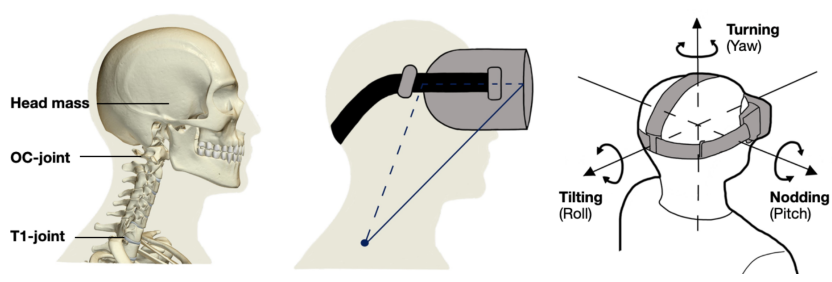

Figure 2: From left to right: anatomical plot of human headneck section, the ball-stick model, three head gestures. 
Nod to Auth also provides visual indicators which instruct users on which head gesture to perform while segmenting out the head gesture data from irrelevant head movements.

\section{PILOT STUDY}

We conducted a pilot study to test the sensing performance of smartphone IMU sensors and the signal fidelity in radius calculation with the ball-stick model. In this study, we used a wood stick with a smartphone attached to a range of positions, simulating different radiuses. We manually rotated the stick with a $180^{\circ}$ rotation angle, and used the collected IMU sensor readings to reconstruct the rotation trajectory based on which we calculated the radius. Details of our algorithm will be described in Section 6.2 and 6.3.

\subsection{Apparatus and Procedure}

We used an Android smartphone (HUAWEI CAZ-TL10) and aligned its screen center to previously marked positions on the stick. The other end of the stick was tied to a fixed pivot point. We tested different radiuses $(10,20,30,40 \mathrm{~cm})$. For each radius, we rotated the stick $180^{\circ}$ (i.e., approximately the maximum angle range for human head) 5 times. The data was streamed to a laptop through TCP/IP for radius calculation.

\subsection{Radius Test}

Figure 3 shows the mean error rate for different wood stick lengths. The results indicate an averaged absolute distance error of $0.33 \mathrm{~cm}$. Overall, longer radius lengths are more prone to error. However, even the largest error is still below $2 \%$ of the total stick length, which we considered as a promising result - it shows enough signal fidelity to disambiguate users we expect to have biometric variances larger than $2 \%$. Additionally, this error could be compensated by leveraging features drawn from other head-neck motion characteristics.

\subsection{Angle Test}

We conducted an offline angle test by selecting data points from a range of angles ( $30^{\circ}$ to $180^{\circ}$ with a $30^{\circ}$ step) centering around center points of rotation trajectories. Figure 4 shows the averaged absolute distance error across angle range. We found that error increases with angle range, caused by the fact that velocity changes more dramatically (i.e., acceleration and deceleration) towards the starting and ending points of trajectories. Some examples can be found in Figure 5, which shows that trajectories towards the ends indeed yield errors higher than those from around the center. Based on this result, we decided to use only the central part of the trajectory, which translates to an angle range of around $20^{\circ}$ of head-neck motions for a more stable radius calculation.

\section{SYSTEM IMPLEMENTATION}

\subsection{Hardware}

Our proof-of-concept AR/VR hardware is based on an Android smartphone (HUAWEI CAZ-TL10) with Google Cardboard. HUAWEI CAZ-TL10 features a built-in BOSCH BMI160 sensor (Accelerometer + Gyroscope) and an AK09911 Magnetometer. We configured the smartphone to stream raw sensor readings and the rotation

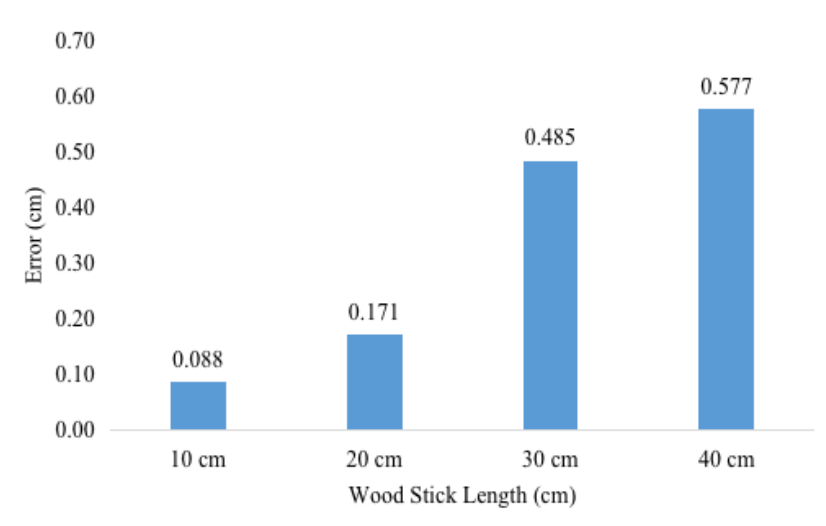

Figure 3: Distance errors of calculated radius across wood stick length (i.e., tested radius).

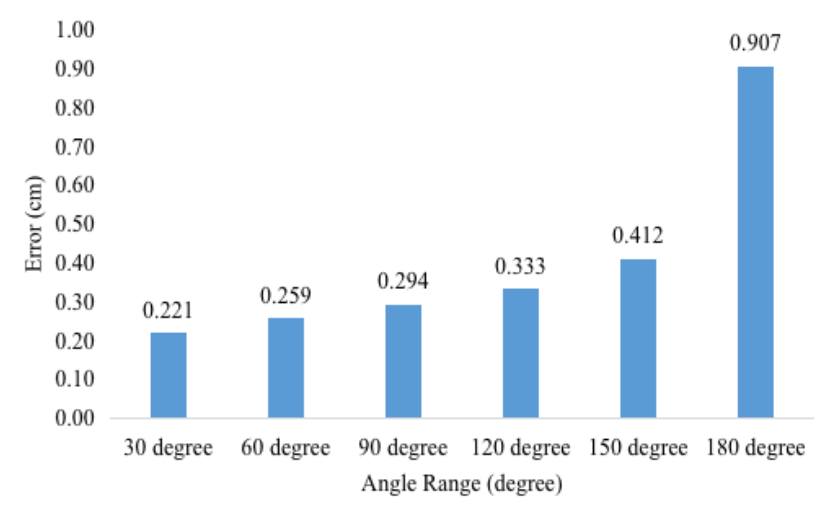

Figure 4: Distance errors of calculated radius across angle.

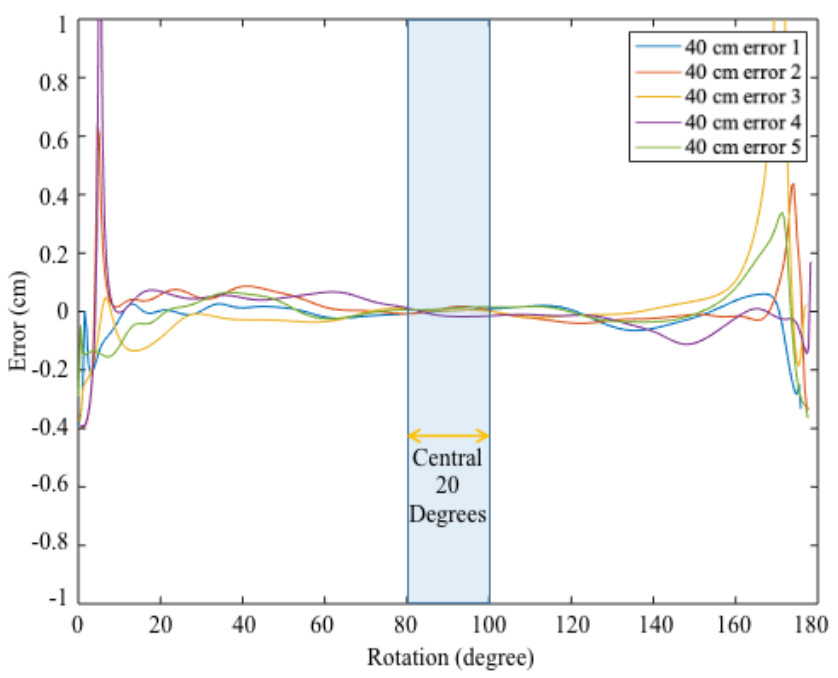

Figure 5: Example error plots.

vector (i.e., Quaternion components along the three axes and a scalar component) to a laptop for further computation at $200 \mathrm{FPS}$. 


\subsection{Signal Processing}

Nod to Auth biometric feature extraction relies on an accurate head trajectory reconstruction, which we discuss next.

6.2.1 Coordinate System Transformation. We first map the linear acceleration of human head rotation from phone coordinates to world coordinates, with a commonly used transformation approach. Below is the rotation matrix derived from the Quaternion provided by the rotation vector.

$$
\begin{gathered}
\mathbf{q}=\left[q_{0}, q_{1}, q_{2}, q_{3}\right]^{T} \\
R(\mathbf{q})=\left[\begin{array}{ccc}
q_{0}^{2}+q_{1}^{2}-q_{2}^{2}-q_{3}^{2} & 2 q_{1} q_{2}+2 q_{0} q_{3} & 2 q_{1} q_{3}-2 q_{0} q_{2} \\
2 q_{1} q_{2}-2 q_{0} q_{3} & q_{0}^{2}-q_{1}^{2}+q_{2}^{2}-q_{3}^{2} & 2 q_{2} q_{3}+2 q_{0} q_{1} \\
2 q_{1} q_{3}+2 q_{0} q_{2} & 2 q_{2} q_{3}-2 q_{0} q_{1} & q_{0}^{2}-q_{1}^{2}-q_{2}^{2}+q_{3}^{2}
\end{array}\right]
\end{gathered}
$$

Assuming that the device and the earth coordinates are denoted as $d$ and $e$ respectively, then a vector expressed in the device frame $\mathbf{v}^{d}$ can be rotated to the earth frame $\mathbf{v}^{e}$ by multiplying the rotation matrix $R(\mathbf{q})[8]$.

6.2.2 Sensor Error Compensation. Errors in acceleration measurements can be amplified through the double integration process, and thus we set to eliminate errors as much as possible early in the process. The acceleration measurements are composed of three main components:

$$
\mathbf{a}=\mathbf{a}_{m}+\mathbf{a}_{g}+\varepsilon
$$

where $\mathbf{a}_{m}$ is the actual acceleration due to head motion, $\mathbf{a}_{g}$ is the acceleration caused by gravity, and $\varepsilon$ is the error which can be decomposed of error plagued by sensor imperfectness, and the computational error resulted from model or calculation inaccuracy during double integration.

We first remove the gravitational acceleration using the default API. To remove non-zero errors when the smartphone is relatively stationary, which could accumulate over time, we used the Zero Velocity Updated algorithm [21, 25, 34] to segment out the motion signals and thus avoid the interference from errors in stationary signals. The main idea of this algorithm is to set a threshold to tell the moving state apart from the stationary period. Since user head movements result in more rotation than translation, we set a threshold on the magnitude of angular speed (i.e., magGyro $=$ $\sqrt{g_{x}^{2}+g_{y}^{2}+g_{z}^{2}}$ where $g_{x}, g_{y}$, and $g_{z}$ are angular velocities along $\mathrm{x}, \mathrm{y}$, and $\mathrm{z}$ axis). To avoid false positives, we adopt a 0.5 -second window buffer - the extracted motion period has to be above the time threshold to trigger the segmentation. Finally, we adopt a 3thorder low-pass digital Butterworth filter to remove high frequency sensor noise.

6.2.3 Head-Neck Motion Trajectory Reconstruction. With the filtered acceleration data from the motion segments, we first derive velocity $\mathbf{v}_{t}$ using the trapezoidal method [1]. To compensate for the non-zero velocity $\mathbf{v}_{e}$ at the end time of the trajectory $T_{e}$, we adopt a common calibration method which subtracts the velocity by an interpolation based on $\mathbf{v}_{e}[1]$ :

$$
\mathbf{v}_{t}^{\prime}=\mathbf{v}_{t}-\mathbf{v}_{e} \frac{t-T_{s}}{T_{e}-T_{s}}, T_{s}<t<T_{e}
$$

Then, we calculate the head-neck motion trajectory $\mathbf{d}$ with the calibrated velocity $\left(\mathbf{v}_{t}^{\prime}\right)$ by

$$
\mathbf{d}_{t}=\mathbf{d}_{t-\Delta t}+\frac{\mathbf{v}_{t}^{\prime}(t-\Delta t)+\mathbf{v}_{t}^{\prime}(t)}{2} \Delta t
$$

\subsection{Feature Extraction and Machine Learning}

We engineered features around user head-neck motion characteristics (i.e., initial-end postures, skeletal proportions, and gesture dynamics) for machine learning. Specifically, we first calculate user head-neck radius based on a circumcircle approach - for every three consecutive points on the central $20^{\circ}$ segment of a motion trajectory, we calculate the radius of their circumcircle. This method yields multiple radius results with which we calculate the mean radius and standard deviation as features. We draw the rest of the features from initial-end postures (i.e., orientation variations orthogonal to the head gesture), and from gesture dynamics which include angular velocity magnitude, properties of trajectory, and power spectral density of the linear acceleration magnitude. Table 1 details all features we engineered. For our proof-of-concept imple-

\begin{tabular}{|c|c|}
\hline Features & Notation and Description \\
\hline Head-Neck Radius & $\begin{array}{l}\text { mean_r: mean rotation radius of the } \\
\text { head-neck motion trajectory } \\
s t d \_r \text { : standard deviation of rotation } \\
\text { radiuses of the head-neck motion tra- } \\
\text { jectory }\end{array}$ \\
\hline $\begin{array}{l}\text { Angular Velocity } \\
\text { Magnitude }\end{array}$ & $\begin{array}{l}\text { mean_gyro: mean of angular velocity } \\
\text { magnitude } \\
\text { std_gyro: standard deviation of angu- } \\
\text { lar velocity magnitude }\end{array}$ \\
\hline $\begin{array}{l}\text { Irrelevant Orientation } \\
\text { Changes }^{1}\end{array}$ & $\begin{array}{l}\text { pitch: degrees of rotation around the } \\
\text { device } \mathrm{x} \text { axis } \\
\text { roll: degrees of rotation around the de- } \\
\text { vice } \mathrm{y} \text { axis } \\
\text { yaw: degrees of rotation around the } \\
\text { device } \mathrm{z} \text { axis }\end{array}$ \\
\hline $\begin{array}{l}\text { Properties of Trajectory } \\
\text { Projections }\end{array}$ & $\begin{array}{l}\text { skewness: skewness of the whole tra- } \\
\text { jectory projection } \\
\text { kurtosis: kurtosis of the whole trajec- } \\
\text { tory projection }\end{array}$ \\
\hline $\begin{array}{l}\text { Power Spectral Density } \\
\text { of Linear Acceleration } \\
\text { Magnitude }\end{array}$ & $\begin{array}{l}\text { mean_psd: average of acceleration } \\
\text { power spectrum density } \\
\text { std_psd: standard deviation of power } \\
\text { spectrum density } \\
\text { skew_psd: skewness of power spec- } \\
\text { trum density } \\
\text { kurt_psd: kurtosis of power spectrum } \\
\text { density }\end{array}$ \\
\hline
\end{tabular}
mentation, we used Random Forest Classifier (by the scikit-learn library, 250 trees) in N-label classification tasks.

Table 1: Extracted features from reconstructed head-neck motion trajectories.

[1] Irrelevant orientation changes are orthogonal to the head gesture, e.g., yaw and roll changes of a Nodding (pitch) head gesture. 


\section{EVALUATION}

\subsection{Participants and Procedure}

We collected data from 10 participants of ages from 23 to 52 ( 5 females). Before the study started, we measured biometric data from participants such as neck length and height. The neck length was measured from the mandibular angle to the mid-portion of the ipsilateral clavicle [13]. We measured an average neck length of $13.2 \mathrm{~cm}(\mathrm{SD}=1.1)$.

To emulate the use scenario of the application Nod to Auth in the real world as much as possible, we included two postures: sitting and standing. Participants first previewed the application and familiarized themselves with our head gestures after putting on the headset. We collected data from six different head motions -2 directions $\times 3$ gestures (Figure 2 right). We recorded 10 trials for each motion from each user ( 5 for sitting, 5 for standing) with a 30-second rest between trials when participants took off the headset and put it back on before the next trial started. In total, we collected 600 data points (i.e., 5 trials $\times 2$ postures $\times 2$ directions $\times 3$ gestures $\times 10$ participants)

\subsection{Training and Classification}

Nod to Auth targets the user authentication among small user groups within for e.g., families, labs, and offices. Therefore, we randomly selected 2, 3, 4, and 5 users from the 10 participants in our study to form groups within which we perform N-label classification tasks. Take a group size of $\mathrm{N}$ for example. There are $C_{10}^{N}$ unique combinations. We collected 10 trials from each participant for each gesture, and thus in this group of size $\mathrm{N}$, we had a total number of $10 \times \mathrm{N}$ trials for each gesture. Among these trials, we used one for testing while training the model with the rest. Our 30-second break in between trials avoids overfitting because no data with time adjacency (i.e., data with unfair similarity) were included in both training and testing sets. Finally, we averaged the accuracies across all combinations across gestures and group sizes. Figure 6 shows the results, which we will discuss in detail next.

\subsection{Results}

7.3.1 User Identification Accuracy. Our result indicates an average accuracy of $98 \%$ ( $\mathrm{SD}=0.01$ ) across all user group sizes and head gestures. The performance slightly declines as the size of the user group increases. Among the three gestures (i.e., nodding, turning, and tilting), the tilting yields the best performance with all accuracies above $98 \%$ regardless of the user group size. The turning gesture performs slightly worse than other gestures, however, none of the performance differences in this section was significant. Additionally, user posture (i.e., standing vs. sitting) is not a significant factor on accuracy.

7.3.2 Error Analysis. Figure 7 shows confusion matrices of the user identification results with the four group sizes we tested, averaged across all gestures. First, all participants achieved over 95\% identification accuracy and we found no statistical outliers. We found that certain pairs of users were more likely to be confused than others. For example, the confusion between User5 and User6 was higher than that between others across almost all user group sizes. By looking into their biometrics (attached in the auxiliary file), we

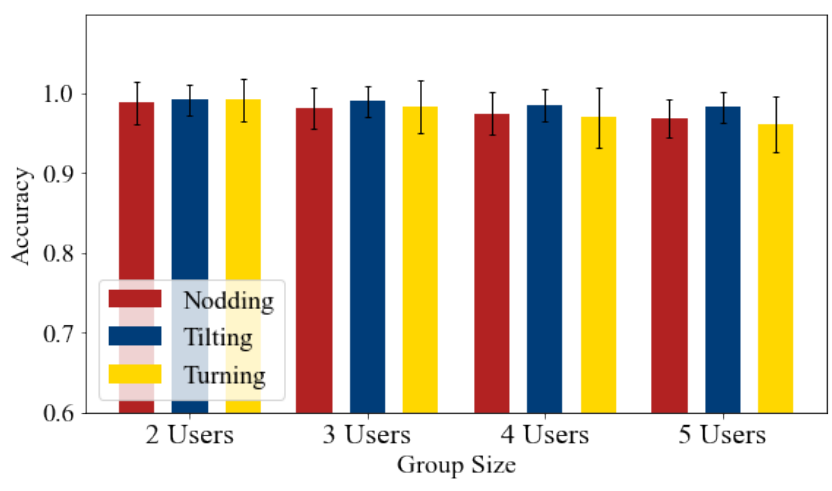

Figure 6: User identification accuracy across user group sizes and head gestures.
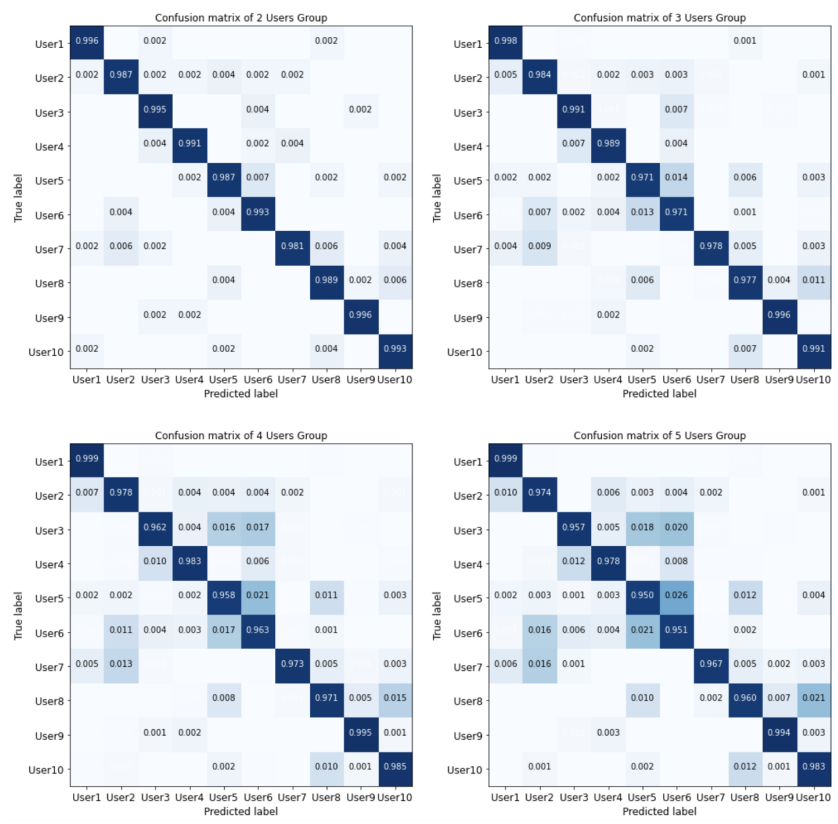

Figure 7: Confusion matrices of various group sizes.

found that these two users shared identical heights and similar neck lengths, which might explain why it is challenging for Nod to Auth to disambiguate between them. A counterexample can be found from User1 and User9 who had very different bio-metrics and thus yielded accuracies of almost $100 \%$. Finally, we did not find age and height to be significant factors on accuracy.

\section{DISCUSSION AND FUTURE WORK}

Nod to Auth achieves encouraging accuracies, which merits further investigation in our future work. First, we want to explore more complex biomechanic systems such as the two-pivot model (Figure 2 left), including those that consider neck's elasticity (i.e., elongation and compression), which have seen success in prior literature $[3,6]$. Second, we will deploy our system in long-term evaluations, which will fully tease out our system's performance especially against user 
variance throughout a day. Finally, there might be opportunities to combine our technique with other sensors on AR/VR devices such as microphones and front cameras for multi-model sensing, which can potentially result in more natural interactions that do not rely on pre-defined gestures with superior performance.

\section{CONCLUSION}

We propose Nod to Auth, a user authentication technique that allows users to quickly unlock their AR/VR devices by single-stroke head gestures. By leveraging the IMU sensors on AR/VR devices, we reconstruct the head-neck motion trajectory, from which we extract machine learning features based on three head-neck motion characteristics (i.e., initial-end postures, skeletal proportions, and gesture dynamics). The results indicate an average accuracy of $97.1 \%$ (over 95\% for all participants). Nod to Auth demonstrates potentials to facilitate AR/VR users with swift and natural authentication, which we hope to further explore in the future.

\section{REFERENCES}

[1] Martin Arraigada and Manfred Partl. 2006. Calculation of displacements of measured accelerations, analysis of two accelerometers and application in road engineering. In 6th Swiss transport research conference. 1.

[2] Musa Atas. 2017. Hand Tremor Based Biometric Recognition Using Leap Motion Device. IEEE Access 5 (2017), 23320-23326. https://doi.org/10.1109/ACCESS.2017. 2764471

[3] Alien C Bosio and Bruce M Bowman. 1986. Simulation of head-neck dynamic response in- Gx and $+G y$. Technical Report. SAE Technical Paper. https://doi. org/10.4271/861895

[4] Attaullah Buriro, Bruno Crispo, Filippo Del Frari, and Konrad S. Wrona. 2016 Hold and Sign: A Novel Behavioral Biometrics for Smartphone User Authentication. In 2016 IEEE Security and Privacy Workshops, SP Workshops 2016, San Fose, CA, USA, May 22-26, 2016. IEEE Computer Society, 276-285. https: //doi.org/10.1109/SPW.2016.20

[5] Cory Cornelius, Ronald A. Peterson, Joseph Skinner, Ryan J. Halter, and David Kotz. 2014. A wearable system that knows who wears it. In The 12th Annual International Conference on Mobile Systems, Applications, and Services, MobiSys'14, Bretton Woods, NH, USA, June 16-19, 2014, Andrew T. Campbell, David Kotz, Landon P. Cox, and Zhuoqing Morley Mao (Eds.). ACM, 55-67. https://doi.org/ $10.1145 / 2594368.2594369$

[6] M De Jager, AAHJ Sauren, J Thunnissen, and JSHM Wismans. 1994. A threedimensional head-neck model: Validation for frontal and lateral impacts. SAE transactions (1994), 1660-1676. https://doi.org/10.4271/942211

[7] Marinus Karel Johannes De Jager. 1998. Mathematical head-neck models for acceleration impacts. (1998).

[8] James Diebel. 2006. Representing attitude: Euler angles, unit quaternions, and rotation vectors. Matrix 58, 15-16 (2006), 1-35

[9] Muhammad Ehatisham-ul-Haq, Muhammad Awais Azam, Jonathan Loo, Kai Shuang, Syed Islam, Usman Naeem, and Yasar Amin. 2017. Authentication of Smartphone Users Based on Activity Recognition and Mobile Sensing. Sensors 17, 9 (2017), 2043. https://doi.org/10.3390/s17092043

[10] Michael Fanton, Calvin Kuo, Jake Sganga, Fidel Hernandez, and David B. Camarillo. 2019. Dependency of Head Impact Rotation on Head-Neck Positioning and Soft Tissue Forces. IEEE Trans. Biomed. Eng. 66, 4 (2019), 988-999. https://doi.org/10.1109/TBME.2018.2866147

[11] Markus Funk, Karola Marky, Iori Mizutani, Mareike Kritzler, Simon Mayer, and Florian Michahelles. 2019. LookUnlock: Using Spatial-Targets for UserAuthentication on HMDs. In Extended Abstracts of the 2019 CHI Conference on Human Factors in Computing Systems, CHI 2019, Glasgow, Scotland, UK, May 04-09, 2019, Regan L. Mandryk, Stephen A. Brewster, Mark Hancock, Geraldine Fitzpatrick, Anna L. Cox, Vassilis Kostakos, and Mark Perry (Eds.). ACM. https://doi.org/10.1145/3290607.3312959

[12] Anhong Guo, Robert Xiao, and Chris Harrison. 2015. CapAuth: Identifying and Differentiating User Handprints on Commodity Capacitive Touchscreens. In Proceedings of the 2015 International Conference on Interactive Tabletops \& Surfaces, ITS 2015, Funchal, Portugal, November 15-18, 2015, Nuno Jardim Nunes, Enrico Costanza, Patrick Olivier, and Johannes Schöning (Eds.). ACM, 59-62. https://doi.org/10.1145/2817721.2817722

[13] Tae Seung Han, Mi Kyeong Oh, Su Min Kim, Hyun Ju Yang, Bum Soon Lee, Soon Yeob Park, and Won Joon Lee. 2015. Relationship between neck length, sleep, and cardiovascular risk factors. Korean fournal of Family Medicine 36, 1 (2015), 10. https://doi.org/10.4082/kjfm.2015.36.1.10
[14] JL Hess and Ch F LOMBARD. 1958. Theoretical investigations of dynamic response of man to high vertical accelerations. The fournal of aviation medicine 29, 1 (1958), 66-75.

[15] Michael Kleinberger. 1993. Application of finite element techniques to the study of cervical spine mechanics. Technical Report. SAE Technical Paper. https: //doi.org/10.4271/933131

[16] Duane Knudson. 2007. Fundamentals of Biomechanics: Second Edition. 24 pages. https://doi.org/10.1007/978-0-387-49312-1

[17] Wei-Han Lee and Ruby B. Lee. 2017. Implicit Smartphone User Authentication with Sensors and Contextual Machine Learning. In 47th Annual IEEE/IFIP International Conference on Dependable Systems and Networks, DSN 2017, Denver, CO, USA, Fune 26-29, 2017. IEEE Computer Society, 297-308. https://doi.org/10.1109/ DSN.2017.24

[18] Sugang Li, Ashwin Ashok, Yanyong Zhang, Chenren Xu, Janne Lindqvist, and Marco Gruteser. 2016. Whose move is it anyway? Authenticating smart wearable devices using unique head movement patterns. In 2016 IEEE International Conference on Pervasive Computing and Communications, PerCom 2016, Sydney, Australia, March 14-19, 2016. IEEE Computer Society, 1-9. https://doi.org/10. 1109/PERCOM.2016.7456514

[19] Guy F Midgley, Ian D Davies, Cécile H Albert, Res Altwegg, Lee Hannah, Gregory O Hughes, Lydia R O'Halloran, Changwan Seo, James H Thorne, and Wilfried Thuiller. 2010. BioMove-an integrated platform simulating the dynamic response of species to environmental change. Ecography 33, 3 (2010), 612-616. https://doi.org/10.1111/j.1600-0587.2009.06000.x

[20] Tahrima Mustafa, Richard Matovu, Abdul Serwadda, and Nicholas Muirhead. 2018. Unsure How to Authenticate on Your VR Headset?: Come on, Use Your Head!. In Proceedings of the Fourth ACM International Workshop on Security and Privacy Analytics, IWSPA@CODASPY 2018, Tempe, AZ, USA, March 19-21, 2018, Rakesh M. Verma and Murat Kantarcioglu (Eds.). ACM, 23-30. https: //doi.org/10.1145/3180445.3180450

[21] Sang Kyeong Park and Young Soo Suh. 2010. A Zero Velocity Detection Algorithm Using Inertial Sensors for Pedestrian Navigation Systems. Sensors 10, 10 (2010), 9163-9178. https://doi.org/10.3390/s101009163

[22] Ken Pfeuffer, Matthias J. Geiger, Sarah Prange, Lukas Mecke, Daniel Buschek, and Florian Alt. 2019. Behavioural Biometrics in VR: Identifying People from Body Motion and Relations in Virtual Reality. In Proceedings of the 2019 CHI Conference on Human Factors in Computing Systems, CHI 2019, Glasgow, Scotland, UK, May 04-09, 2019, Stephen A. Brewster, Geraldine Fitzpatrick, Anna L. Cox, and Vassilis Kostakos (Eds.). ACM, 110. https://doi.org/10.1145/3290605.3300340

[23] Nurizal Dwi Priandani, Herman Tolle, and Fitri Utaminingrum. 2017. Real Time Advanced Head Move-ment Recognition for Application Controller Based On Android Internal Gyroscope Sen-sor. Int. F. Adv. Soft Comput. Its Appl 9, 1 (2017).

[24] Munehiko Sato, Rohan S. Puri, Alex Olwal, Yosuke Ushigome, Lukas Franciszkiewicz, Deepak Chandra, Ivan Poupyrev, and Ramesh Raskar. 2017. Zensei: Embedded, Multi-electrode Bioimpedance Sensing for Implicit, Ubiquitous User Recognition. In Proceedings of the 2017 CHI Conference on Human Factors in Computing Systems, Denver, CO, USA, May 06-11, 2017, Gloria Mark, Susan R. Fussell, Cliff Lampe, m. c. schraefel, Juan Pablo Hourcade, Caroline Appert, and Daniel Wigdor (Eds.). ACM, 3972-3985. https://doi.org/10.1145/3025453.3025536

[25] R. P. Suresh, V. Sridhar, J. Pramod, and V. Talasila. 2018. Zero Velocity Potential Update (ZUPT) as a Correction Technique. In 2018 3rd International Conference On Internet of Things: Smart Innovation and Usages (IoT-SIU). 1-8. https://doi. org/10.1109/IoT-SIU.2018.8519902

[26] Chieh S Tien and Ronald L Huston. 1985. Biodynamic modelling of the head/neck system. Technical Report. SAE Technical Paper. https://doi.org/10.4271/850438

[27] Pedro Tome-Gonzalez, Julian Fiérrez, Rubén Vera-Rodríguez, and Mark S. Nixon. 2014. Soft Biometrics and Their Application in Person Recognition at a Distance. IEEE Trans. Inf. Forensics Secur. 9, 3 (2014), 464-475. https://doi.org/10.1109/TIFS. 2014.2299975

[28] Waqas Wazir, Hasan Ali Khattak, Ahmad Almogren, Mudassar Ali Khan, and Ikram Ud Din. 2020. Doodle-Based Authentication Technique Using Augmented Reality. IEEE Access 8 (2020), 4022-4034. https://doi.org/10.1109/ACCESS.2019. 2963543

[29] JL Williams and TB Belytschko. 1983. A three-dimensional model of the human cervical spine for impact simulation. (1983). https://doi.org/10.1115/1.3138428

[30] JSHM Wismans, H Van Oorschot, and HJ Woltring. 1986. Omni-directional human head-neck response. SAE transactions (1986), 819-837. https://doi.org/10. $4271 / 861893$

[31] Guannan Wu, Jian Wang, Yongrong Zhang, and Shuai Jiang. 2018. A Continuous Identity Authentication Scheme Based on Physiological and Behavioral Characteristics. Sensors 18, 1 (2018), 179. https://doi.org/10.3390/s18010179

[32] Jonathan Wu, Prakash Ishwar, and Janusz Konrad. 2014. The value of posture, build and dynamics in gesture-based user authentication. In IEEE International foint Conference on Biometrics, Clearwater, IFCB 2014, FL, USA, September 29 October 2, 2014. IEEE, 1-8. https://doi.org/10.1109/BTAS.2014.6996261

[33] Shanhe Yi, Zhengrui Qin, Edmund Novak, Yafeng Yin, and Qun Li. 2016. GlassGesture: Exploring head gesture interface of smart glasses. In 35th Annual IEEE 
International Conference on Computer Communications, INFOCOM 2016, San Francisco, CA, USA, April 10-14, 2016. IEEE, 1-9. https://doi.org/10.1109/INFOCOM. 2016.7524542

[34] Rui Zhang, Hai Yang, Fabian Höflinger, and Leonhard M Reindl. 2017. Adaptive zero velocity update based on velocity classification for pedestrian tracking. ieee Sensors journal 17, 7 (2017), 2137-2145. https://doi.org/10.1109/JSEN.2017.
2665678

[35] Yongpan Zou, Meng Zhao, Zimu Zhou, Jiawei Lin, Mo Li, and Kaishun Wu. 2018. BiLock: User Authentication via Dental Occlusion Biometrics. Proc. ACM Interact. Mob. Wearable Ubiquitous Technol. 2, 3 (2018), 152:1-152:20. https: //doi.org/10.1145/3264962 